\title{
THE PGNN FOR THE DIFFERENTIATION OF SYNDROMES OF THE KIDNEY
}

\author{
Yun $\mathrm{Wu}^{1}$ Changle Zhou ${ }^{2}$ Zhifeng Zhang ${ }^{3}$ \\ 'Institute of Artificial Intelligence, XiaMen University, FuJian XiaMen 361005, China \\ \{E-mail: yininwu,a $126.00 \mathrm{~m}$ \} \\ ${ }^{2}$ Institute of Artificial Intelligence, XiaMen University, FuJian XiaMen 361005, China \\ \{E-mail: dozero(axmu.edu.cn\} \\ ${ }^{3}$ Shanghai University of Traditional Chinese Medicine, ShangHai 201203, China \\ \{E-mail: rchbto163.com\}
}

\begin{abstract}
The research into the impersonality and information of the Traditional Chinesemedicine Diagnosis (TCMD) is recognized to be a crucial work, especially for the Traditional Chinese-medicine (TCM) medicine examination system. However the tongue is like a mirror of the Viscera, and the pathological changes of them can reflect on the tongue. So according the information of the Sizhen for the syndromes of the Kidney, we design the initial structure of the PGNN(Probabilistic Genetic Neural Network), and its connection weight and the structure of the PGNN automatically will be optimized by the GA and others optimal way: It is certain that the groping research will be use for the modemization of the TCM.
\end{abstract}

Keywords: TCMD (Traditional Chinese Medical Diagnosis); Differentiation of Syndromes of the Viscera; Syndromes of the Kidney; PGNN; Genetic Algorithm (GA).

\section{INTRODUCTION}

Sizhen is the crucial way to collect information for the TCM diagnosis, specially the inspection of the tongue, which is the symptom information. Because in the clinic it has been proved that the symptom of the tongue changed rapidly and brilliantly during the developing of the diseases. It is like a mirror of the Viscera, and the pathological changes can reflect on the tongue, namely the symptom information of the tongue is changed. So it

Please use the following format when citing this chapter:

Wu, Y, Zhou, C, Zhang, Z, 2006, in IFIP International Federation for Information Processing, Volume 228, Intelligent Information Processing III, eds. Z. Shi, Shimohara K., Feng D., (Boston: Springer), pp. 337-345. 
becomes the important evidence for the TCMD. But in fact, only using the symptom information of the tongue to diagnose the Syndromes of the Viscera is not enough, so some others common manifestations are also used auxiliarily.

Many successful medicine systems are based on the theory of the western medicine, which uses the symbol processing method to express the medicine information. It is mostly because the computer can process the formalized information of the western medicine. But this way does not suit the TCMD, which information is fuzzy, incomplete and unclear. If we also use the symbol processing method to express the information of the TCMD, it will easily lose much important information, and lots of information can not be processed, which makes the system has low validity.

So the TCM medical examination system which we design integrates some Al technologies, such as logic, ANN (Artificial Neural Network), GA, and others soft computing. The main theories are: First according the characteristics of the manifestation, and get the logical rule. Second construct the ANN for every kind of the TCMD diagnosis. The common ANN has very weak automaticity, which can not satisfy the variety of the TCM clinic, so using the Probabilistic GNN to construct the TCMD NN database. In the practices, the TCM medical examination system will select ANN form TCMD ANN database to compute the diagnosis result. So the computing capability of the TCMD ANN is the most important knowledge database to the TCM medical examination system.

This paper is organized as follows. In section 2, we mainly present the PGNN, which base on the symptoms of tongue and others common information for the differentiation of syndromes of the Kidney. In section 3 , we introduce the improved GA to optimize the PGNN, which forms are presented in section 2. In section 4, the test results are discussed. The conclusions and the future work are disused in section 5 .

\section{THE PGNN FOR THE DIFFERENTIATION OF SYNDROMES OF THE KIDNEY}

\subsection{The structure of the PGNN for the Differentiation of Syndromes of the Kidney}

The initial PGNN is a forward NN, which consists of three layers. The first layer is the input layer ( the $x_{i} \in[0,1]$ ), generally consisting of sixty neural cells determined by the symptom manifestations. The second layer is the hidden layer, which is determined initially by our experience, but finally is determined by input training set. The last layer is the output layer, which has 
generally the same size as the syndromes which need to be diagnosed by the PGNN. The initial structure is shown in figurel.

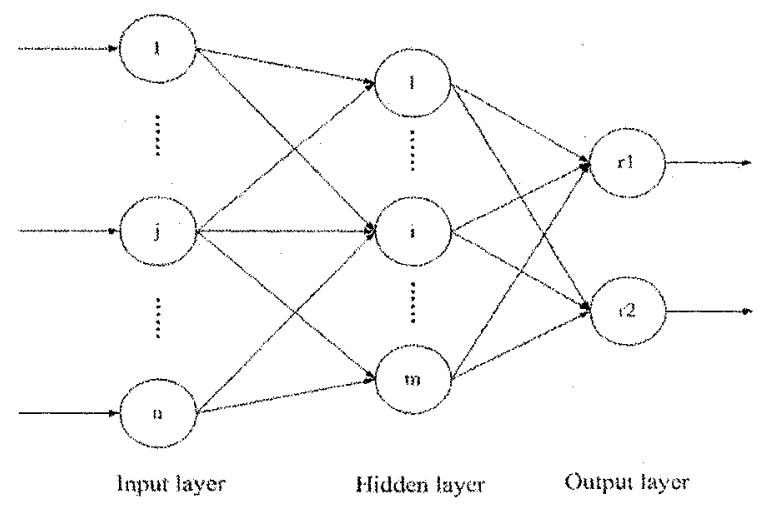

Figure 1, the three-layer PGNN's structure

\subsection{The region division of the tongue}

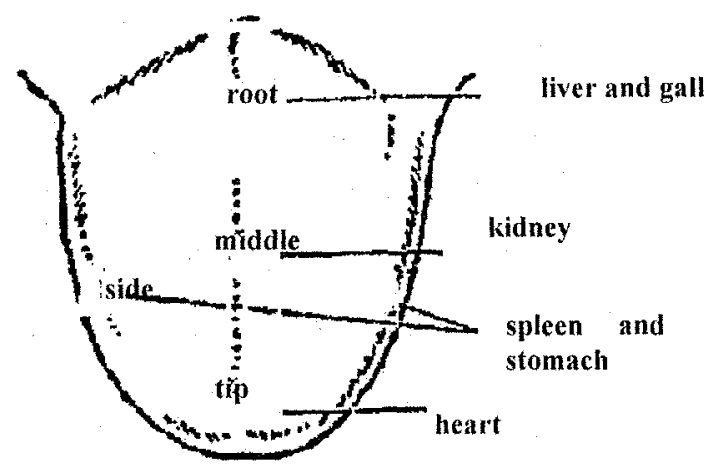

Figure 2. the division of the tongue body

During clinical practice, human discover the pathological changes of the bowels can reflect to the change of the tongue features. So it has been formed the dissertation that the pathological changes of the viscera will be reflected on the tongue, and the pathological changes of the different viscera organs will reflect on different subareas of the tongue. Usually the tongue would be divided five subareas ${ }^{[1]}$, as show in picture 2, according to the symptoms on the different subareas, the diagnosis system will select different ANN from the diagnosis ANN database. The sides of the tongue 
reflect the pathological changes of the liver and gall. The tip of the tongue reflects the pathological changes of the heart. The middle of the tongue reflects the pathological changes of the spleen and stomach. The root of the tongue reflects the pathological changes of the kidney. So if some viscera organs have pathological changes, some symptoms will appear on the subareas of the tongue. So if the kidney has some disease, the root of the tongue will have some unusual symptom manifestations.

\subsection{The symptoms information of the Differentiation of Syndromes of the Kidney}

The symptom manifestations of the tongue are sorted to thirty-one, such as: The color of the tongue body: light white; light red; crimson; bluish purple. The shape quality of the tongue body: tender; old; fat; thin; fissured; prickled; emaciated; petechia; canker; Teeth-printed.

The state of the tongue: wry; tremulous; stiff; shortened; flaccid.

The color of the coating: white; yellow; gray-black.

The color of the tongue vessel: light color; deep color.

The proper of the coating: thin; thick; moist; dry; greasy; crude; exfoliative; These informations of the tongue are very important to diagnose the syndrome. For example, if the color of the root of the tongue body is yellow, the kidney maybe has the hot syndrome. So each symptom like that is an input neural cell in the Probabilistic GNN. This information is summed up form the clinic case of the Shanghai University of Traditional Chinese Medicine and the Hospital of Taicang, which are our cooperators.

\subsection{Others manifestation of symptoms}

In order to improve the validity of the diagnosis, some others common symptom manifestations are added, which are auxiliary informations to differentiate the Syndrome of the Kidney Disease. Such as, Interrogation informations: fearing cold; fearing heat; the pain (such as head; chest; coerce; stomach; abdomen; waist; extremity, est.); the state of the head and the body (such as swirling; swimming; thin; fat, est.).

For the sake of confirming the correction of the diagnosis, those informations are also necessary.

\subsection{The PGNN}

According the symptoms manifestations of the patient, the Probabilistic NN differentiates the syndrome can be simply abstract to this formula:

$$
f(R \times C \times D) \rightarrow p\left(x_{1}\right) \times p\left(x_{2}\right) \times \cdots \times p\left(x_{n}\right)
$$


The $\mathrm{R}$ means the set of the subareas of the tongue body; the $\mathrm{C}$ means the set of all the unusual symptom manifestations of the tongue; the D means the set of all the others unusual symptom manifestations. $f$ is a value function that is used to compute the strong-wreak degree of the syndrome's appearing. The $x_{1}, x_{2}, \ldots, x_{n}$ means there are $n$ syndromes is needed to diagnose, the $0 \leq p\left(x_{i}\right) \leq 1$ is the appearing probability of the $x_{i}$ syndrome. So in here, the probability of the symptom manifestations in the sets of the $R, C$ and $D$ is the input value of the Probabilistic NN, the $p\left(x_{i}\right)$ is the output value of the Probabilistic GNN, the $f$ is just the Probabilistic NN. According the experience of the TCM expert, there is a threshold $\alpha$ is needed, which compare with the $p\left(x_{i}\right)$. If $\alpha \leq p\left(x_{i}\right)$, the $x_{i}$ syndrome maybe appear, else it maybe not. At the same time, in order to make the Probabilistic NN is the best for the CTMD, the structure and the link-value of the Probabilistic NN is optimized by the Genetic Algorithm, which is the Probabilistic GNN (PGNN).

\section{THE OPTIMIZING ALGORITHM}

The main steps of the GA for optimizing the PGNN are:

(1). Hybrid encoding. The neural network (PGNN) is written to one realvalued two-matrix and one binary-valued two-matrix. The real-valued matrix shows the link-weights of the network. The other matrix shows the validity of the link between neurons and the link-weights, which is propitious to adjust the construction of the PGNN.

(2). Initialization. Randomly generate an initial population of the PGNN, which has the characters talked above. Before next step, each network is partially trained, which can avoid overstraining. At the same time, given the error value $\delta>0$.

(3). Fitness function. The stylebook data are divided into two sets, namely training set $(\mathrm{T})$ and validation set $(\mathrm{V})$, which will be adjusted properly during the computing process. After partially trained, calculate the fitness of each PGNN. The fitness function is:

$$
\begin{gathered}
f(x)=\alpha \times E(T)+\beta \times D(N)+\gamma \times L(N) \\
E(T)=\frac{1}{M S E(x)}
\end{gathered}
$$

The $D(N)$ is the number of the hidden neural cells. The $L(N)$ is the number of the links. It means that the PGNN whose size is small is better than whose size is big, even if they have the same $E(T)$. The $\alpha, \beta$ and $\gamma$ are fixed parameter. 
(4). Genetic operation. In order to keep the good genes, $\mathrm{K}$ individuals whose fitness is good are directly copied to next generation. The others offspring comes from these operators.

(1) Selection: According the fitness, the ith individual is selected with such probabilistic of

$$
p_{1}=\frac{f\left(x_{i}\right)}{\sum_{i=1}^{m} f\left(x_{i}\right)}
$$

If the $\eta<p_{i}$, the individual is selected. The $\eta$ is a random number.

(2) Crossover. In order to avoid the permutation, this is a difficult resolved problem in the structure adjustment. It means that after the crossover operation, the structure of the PGNN maybe became invaluable. So in this algorithm, the crossover operation has been canceled.

(3) Mutation. Select individuals and conduct mutations according to the current mutation rate $p_{m}$. Considering the method of the case learning which are always applied in the TCM expert systems, so the mutation uses the thought of PSO ${ }^{[4]}$, namely according the fitness of the best individual and the worst individual, randomly select two individuals, such as $i$ and $j$, then mutation is implemented as follows:

$$
x_{i, j}^{\prime}=x_{i, j}+\phi_{1} \times\left(f\left(p_{g}(t)\right)-f\left(x_{i, j}\right)\right)+\phi_{2} \times\left(f\left(p_{d}(t)\right)-f\left(x_{i, j}\right)\right)
$$

the $p_{g}(t)$ is the best evolutionary PGNN of the all generation, $p_{d}(t)$ is the best evolutionary PGNN of this generation, $\phi_{1}, \phi_{2} \in[-1,1]$ are random number. This method simulates the thought of TCM case learning, and is propitious to the convergence of the GA.

(5). Structure adjustment. If those operations can not prove the calculating capability of the PGNN, then adjust its structure. They include four operators. The first one is deleting one link between two hidden neural cells. The second is deleting on hidden neural cell, so the links between the deleted cell and others hidden cell are also delete. The third is adding one link between two hidden cells. And at last it is adding one hidden neural cell. As usually, the deleting operation is preceded the add operation, which is benefit for keeping the structure of the PGNN is small. In one time, as long as one operation works, the others operators do not be used and return to make a new computing.

(6). Ending condition. If the maximal evolutionary epoch is reached or the error value is less than the $\alpha$, the optimizing process is over. 


\section{DISCUSSION}

The test data come from the Shanghai University of Traditional Chinese Medicine and the Hospital of Taicang. So in the test-data, there are hundred data come from the reference book[1], fifty clinical data(the Deficiency of the Kidney-yang or the Deficiency of the kidney-yin) comes form the Shanghai University of Traditional Chinese Medicine and the Hospital of Taicang. In next works, we will increase the clinical data to test it. In the optimizing process, the error value is shown as Figure 3.

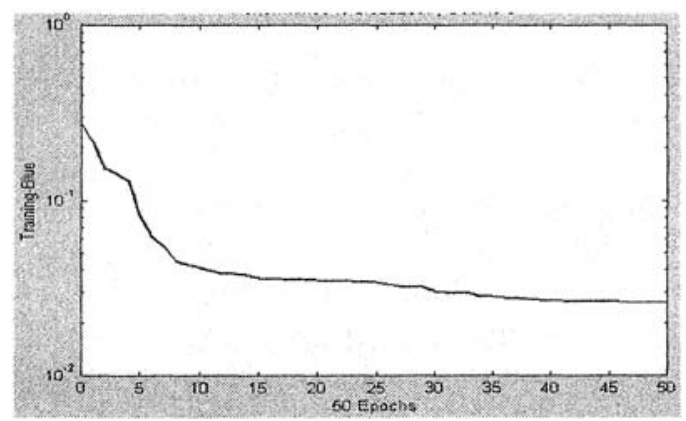

Figure 3. the training graph.

After learning the clinical data, the diagnosis result of the PGNN could be consist with the clinic cases, which in the $T$ set, but to the clinic cases in the $\mathrm{S}$ set, the correct ratio is about $70 \%$. But not stable. however the PGNN exhibits the capability to distinguish the Deficiency of the Kidney-yang and the Deficiency of the kidney-yin, if the patient has the clinical manifestations of the Deficiency of the Kidney-yang or the Deficiency of the kidney-yin, such as lassitude in the loins and knees, cold limbs, feeling listless and inert; swollen tongue with white fur. If the probability of these clinical manifestations is more than $60 \%$ and there are not any other conflict information, the PGNN can diagnosis the patient who has the Deficiency of the Kidney-yang. So if the PGNN can learn more useful information form the clinical cases, it will be more stabile. But the computing time will be up. 


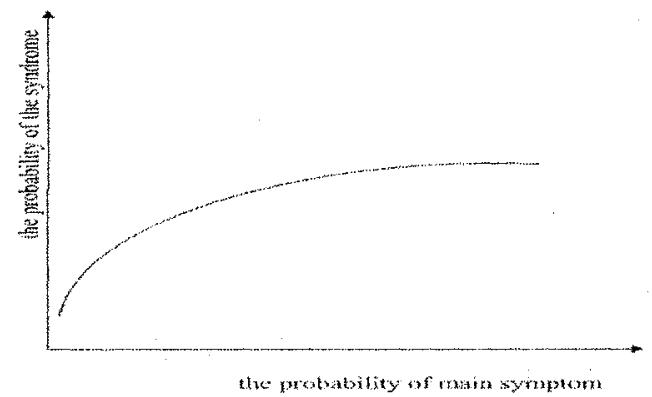

Figure 4. the approximate relationship between of the syndrome and the single main symptom.

As shown in figure 4 , a disease will appear some symptom with high probability. And the probability of the symptom is higher, the ratio of the syndrome is higher. But following the probability of the symptom up, the ratio of the syndrome stop going up (it is concluded form the experiment result, which adjust the probabilistic of one main symptom and only a trend line). For example the white tongue coating is the main symptom of the Deficiency of the Yang on tongue. If we increase the input probabilistic value, the probability of the Deficiency of the Yang will be up. But if we continue to increase the value, the value of the output will be stop to up. So in some degree, it makes us sure that if we can correctly conclude the main symptom of the syndrome, the PGNN can resolve some TCMD computing problems, which maybe still has some subjectivity.

On the other side, the PGNN also can better process some problems than the traditional symbol technique, such as indeterminable information; lowefficiency learning capability, and the difficulty to manage the information $\mathrm{DB}$, which is decided by the characteristic of the PGNN. And it also can automatically learn information from the training data to adjust itself, which lowers these demerits, such as the TCM expert's knowledge is deficient. And can prove the automaticity, reliability, validity and the intelligence of the TCM medicine examination system.

\section{CONCLUSION}

This paper discusses the PGNN, which is used to TCMD-D the Differentiation Syndromes of the Kidney based on the Sizhen information, especially the symptom of the tongue. And after optimizing the weight value and the structure of the PGNN by the GA, the PGNN has better performance 
than common $\mathrm{NN}$. In some degreed, it can diagnose the viscera syndromes correctly, but in some clinic data, it difficultly differentiate the syndromes, specially the clinic manifestations is not evidence, even if there are some conflict or faintness symptoms manifestations appear on the patient. So it need to add others technique to solve these information-Fuzzy technique, and some others optimized ways, which is our next research works.

\section{REFERENCES}

1. Wenfeng Zhu, Traditional Chinese Medicine Diagnostics [M]Beijing : Chinese Medicaments Press, 2004.

2. Nenggan Zhang, TCM-SIRD:An Intergrated Aided System for Traditional Chinese Medicine Sizheng[I], 2004 IEEE International Conference on System, Man and Cybernetics, 2004, (3864-3868)

3. Xuezhong Zhou, TCMMDB A Distributed MultiDatabase Query System and It's Key Technique Implement, 2004, IEEE(1095-1098)

4. Chia-Feng Juang, On The Hybrid of Genetic Algorithm and Particle Swarm Optimization For Evolving Recurrent Neural Network, 2004, IEEE (2285-2288)

5. Z.-H Tan , Hybrid evolutionary approach for designing neural networks for classification, ELECTRONICS LETTERS 22nd July 2004 Vol. 40 No. 15

6. Xu Ning, Neural Network Based Expert System for Palmprint Diagnosis, Compute Application and Research, 2001. Vol.4-6.

7. Yun Wu, Genetic Neural Network for Traditional Chinese Medicine, 2005 international Conference on Neural Networks and Brain, 2005, Vol.339-342 